\title{
Efficacy of Additional Surgical Resection After Endoscopic Submucosal Dissection for Superficial Esophageal Cancer
}

\author{
MICHIHIRO KUDOU ${ }^{1}$, ATSUSHI SHIOZAKI ${ }^{1}$, HITOSHI FUJIWARA ${ }^{1}$, HIROTAKA KONISHI ${ }^{1}$, \\ KATSUTOSHI SHODA ${ }^{1}$, TOMOHIRO ARITA ${ }^{1}$, TOSHIYUKI KOSUGA ${ }^{1}$, RYO MORIMURA ${ }^{1}$, \\ YASUTOSHI MURAYAMA ${ }^{1}$, YOSHIAKI KURIU ${ }^{1}$, HISASHI IKOMA ${ }^{1}$, TAKESHI KUBOTA ${ }^{1}$, \\ MASAYOSHI NAKANISHI ${ }^{1}$, KAZUMA OKAMOTO ${ }^{1}$, OSAMU DOHI ${ }^{2}$, \\ HIDEYUKI KONISHI $^{2}$, YUJI NAITO ${ }^{2}$ and EIGO OTSUJI ${ }^{1}$ \\ ${ }^{I}$ Division of Digestive Surgery, Department of Surgery, Graduate School of Medical Science, \\ Kyoto Prefectural University of Medicine, Kyoto, Japan; \\ ${ }^{2}$ Department of Molecular Gastroenterology and Hepatology, Graduate School of Medical Science, \\ Kyoto Prefectural University of Medicine, Kyoto, Japan
}

\begin{abstract}
Background/Aim: This study aimed to investigate the efficacy of additional surgical resection (ASR) after endoscopic submucosal dissection (ESD) for superficial esophageal cancer (SEC). Patients and Methods: Clinicopathological features and prognoses were analyzed in esophageal cancer (EC) cases with the indication for additional treatments (AT) after ESD (37 cases) and in cases that underwent primary resection (13 cases). Results: Sixteen out of 37 cases underwent ASR. The remaining 21 cases underwent other treatments or observation. Although all ASR cases are alive without recurrence, recurrence developed in 2 non-ASR cases. Residual tumors were detected in 2 ASR cases. All 4 cases were $T 1 b$ and positive for lymph vessel invasion $(l y+)$. No significant difference was observed in surgical outcomes between ASR and primary surgical resection (PSR) cases. Conclusion: AT need to be considered for $T 1 b$ and ly+ cases, and ASR is one of the effective AT. The safety of ASR was similar to PSR for SEC.
\end{abstract}

Recent advances in techniques to diagnose esophageal cancer (EC) have resulted in increases in the frequency of detection of superficial EC. This early detection contributes to the long-term survival of EC patients. Although esophagectomy

Correspondence to: Ass. Prof. Atsushi Shiozaki, Division of Digestive Surgery, Department of Surgery, Kyoto Prefectural University of Medicine, 465 Kajii-cho, Kamigyo-ku, Kyoto 6028566, Japan. Tel: +81 752515527, Fax: +81 752515522, e-mail: shiozaki@koto.kpu-m.ac.jp

Key Words: Superficial esophageal cancer, endoscopic submucosal dissection, additional esophagectomy. with lymph node dissection is regarded as the most curative therapy for EC, the development of more minimally-invasive therapies are needed because anatomical complexity around the esophagus increases the rate of serious complications and reduces quality of life (QOL), e.g. hoarseness and eating disorders, following curative esophagectomy. Endoscopic submucosal dissection (ESD) has advantages over curative esophagectomy, such as organ preservation, the maintenance of QOL, and lower mortality and morbidity rates $(1,2)$. Therefore, endoscopic ESD has been attracting increasing attention as a minimally invasive curative treatment for superficial EC.

The Japanese guidelines for EC described the indication for ESD as invasion to the lamina propria mucosa, while the relative indication for ESD was invasion to the muscularis mucosa (T1a-MM) and minimal invasion to the submucosa (T1b-SM1) (3). Based on these guidelines, a large number of superficial EC patients in Japan now undergo ESD. EC has a high potential for lymph node metastasis, and sometimes occurs in T1a-MM and T1b-SM1 cases. Therefore, some cases of T1a-MM and T1b-SM1 EC cannot be resected completely by ESD, and additional lymph node dissection or other treatments are indispensable as curative treatments for these cases. However, standard additional treatments and criteria for these cases have not yet been established. Controversy surrounds how EC cases diagnosed with T1a-MM or T1b tumors by the pathological findings of ESD specimens are to be additionally treated in order to avoid the recurrence of EC.

In our division, additional esophagectomy with lymph node dissection has been performed for EC cases with potential lymph node metastasis or residual tumors after ESD. The pathological findings of ESD specimens revealed 
residual tumors in some cases. Meanwhile, the recurrence of lymph node metastasis occurred in cases that received nonsurgical additional treatments or observation. These findings suggested the importance of additional treatments after ESD for these cases. This study aimed to investigate the clinical outcomes of EC cases with potential lymph node metastasis after ESD, and the surgical safety of additional esophagectomy after ESD.

\section{Patients and Methods}

Patients. Between January 2008 and December 2015, 37 cases were indicated for additional treatments based on the pathological findings of EC specimens resected by ESD. The indication for additional treatments after ESD was decided based on the Japanese guidelines for EC (3). Briefly, the criteria of additional treatments were as follows. In EC cases with an invasion to the muscularis mucosae based on the pathological findings of ESD specimens, positive venous or lymph vessel invasion, infiltration pattern C (INF C), which indicates cancer nests exhibiting infiltrative growth and an unclear, and droplet infiltration pattern (DI) $(4,5)$ were regarded as indications for additional treatments. Moreover, EC cases diagnosed with invasion to the submucosa or greater and a positive vertical margin were similarly regarded as indications. Sixteen of these cases underwent additional esophagectomy with lymph node dissection after ESD. Forty-nine superficial EC cases without lymph node metastasis underwent primary esophagectomy with lymph node dissection during the study period.

Pathological evaluation and classification. Resected specimens were microscopically examined by at least two experienced pathologists, and evaluated according to the Japanese Classification of Esophageal Cancer, 10th edition $(6,7)$. Briefly, mucosal, submucosal, and muscularis propria cancers were defined as T1a, $\mathrm{T} 1 \mathrm{~b}$, and $\mathrm{T} 2$, respectively. T1a and $\mathrm{T} 1 \mathrm{~b}$ cancers were divided into three subtypes according to the extent of invasion, i.e. T1a-EP: mucosal epithelium, T1a-LPM: lamina propria mucosae, T1a-MM: muscularis mucosa, T1b-SM1: upper-third stratum of the submucosal layer, T1b-SM2: middle-third stratum of the submucosal layer, and T1b-SM3: lower-third stratum of the submucosal layer.

Surgery. Sixteen cases underwent additional esophagectomy after ESD. Transthoracic esophagectomy was performed on 3 of these cases $(18.7 \%)$ and video-assisted thoracic esophagostomy on 1 $(6.2 \%)$. Twelve cases $(75.0 \%)$ underwent esophagectomy with a laparoscopic transhiatal approach. All cases underwent two-field (thoracic and abdominal) lymphadenectomy. Reconstruction after surgery was performed with a gastric tube via the post-sternal route. In 49 cases that underwent primary resection, transthoracic esophagectomy was performed on $25(51.0 \%)$, video-assisted thoracic esophagostomy on $11(22.4 \%)$, and esophagectomy with a laparoscopic transhiatal approach on 13 (26.5\%).

Postoperative complications. Postoperative respiratory complications were defined as those involving major respiratory insufficiency; such as a need for reintubation or severe pneumonia. The motion of the vocal cords was evaluated using laryngeal fibers 1 week after surgery. Recurrent nerve paralysis was diagnosed based on this finding by an experienced otolaryngologist. Surgical site infection was defined using CDC criteria.

Statistical analysis. Comparisons were performed between both groups using the Student's $t$-test, chi-squared test, or Fisher's exact test. Survival curves were estimated by the Kaplan-Meier method, and differences in survival were examined using the Log-rank test. $p$-values of less than 0.05 were regarded as significant. Statistical analyses were performed using JMP version 10 .

\section{Results}

Patient characteristics of EC cases with the indication for additional surgical resection (ASR). Sixteen out of 37 cases with the indication for additional treatments underwent ASR. In the remaining 21 cases, 2 received adjuvant chemotherapy, 7 received adjuvant chemo-radiotherapy, and 12 underwent careful observation. The characteristics of ASR and non-ASR cases were summarized in Table I. No significant differences were observed in gender, pathological invasion depth, lymph vessel invasion, or the type of infiltration between the ASR and non-ASR groups. The ASR group was significantly younger than the non-ASR group $(p=0.001)$. Moreover, all esophageal adenocarcinoma cases underwent $\operatorname{ASR}(p=0.015)$.

Prognoses of ASR and non-ASR cases. We performed a comparative analysis of overall survival (OS), causespecific survival (CSS), and relapse-free survival (RFS) among ASR and non-ASR cases. The 5-year OS rates of ASR and non-ASR groups were $100 \%$ and $76.3 \%$, respectively (Figure $1 \mathrm{~A}$ ), and were not significantly different $(p=0.318)$. Similarly, no significant difference was observed in CSS rates (Figure 1B). One non-ASR case died due to the progression of EC. The 5-year RFS rates of the ASR and non-ASR groups were $100 \%$ and $84.4 \%$, respectively (Figure $1 \mathrm{C}$ ), and were not significantly different $(p=0.336)$. Although no significant differences were observed in the prognosis of the ASR and non-ASR groups, recurrence occurred in 2 non-ASR cases. This result suggests that ASR contributed to the prevention of recurrence among these cases.

Patient characteristics and pathological findings of surgically resected specimens in ASR cases. The reason for ASR and pathological findings in ASR cases are summarized in Table II. The reason for the indication for ASR was lymph vessel invasion in 10 ASR cases, INF C in 2, DI in 5, and a positive vertical margin in 2 . Sixteen cases underwent subtotal esophagectomy with two-field lymph node resection, while 2 underwent middle and lower esophagectomy with two-field lymph node resection. A residual initial tumor was detected in 2 ASR cases (Cases 6 and 12). Although a residual tumor was not detected in case 
Table I. Background of additional surgical resection and non-additional surgical resection cases.

\begin{tabular}{llccc}
\hline Variable & Male & $\begin{array}{c}\text { ASR } \\
(\mathrm{n}=16)\end{array}$ & $\begin{array}{c}\text { non-ASR } \\
(\mathrm{n}=21)\end{array}$ & $p$-Value \\
\hline Gender & Female & 16 & 19 & 0.200 \\
Age & Mean $( \pm$ SD $)$ & $63.1( \pm 7.8)$ & $71.5( \pm 7.1)$ & 0.001 \\
Histological type & AC & 4 & 0 & 0.015 \\
& SCC & 12 & 21 & \\
ESD-T & T1a-MM & 9 & 9 & 0.414 \\
pT & T1b & 7 & 12 & \\
& T1a-MM & 9 & & \\
ESD-ly & T1b & 6 & & \\
& T3 & 1 & & \\
ESD-v & Positive & 10 & 8 & 0.102 \\
ESD-INF & Negative & 6 & 13 & \\
& Positive & 4 & 4 & 0.736 \\
ESD-HM & Negative & 12 & 17 & \\
& C & 2 & 0 & 0.096 \\
ESD-VM & DI & 5 & 9 & 0.471 \\
& Negative & 15 & 21 & 0.354 \\
Additional treatment & Positive & 1 & 0 & \\
& Negative & 14 & 17 & 0.404 \\
& Positive & 2 & 4 & \\
& Surgery & 16 & 0 & \\
& CT & 0 & 2 & \\
& CRT & 0 & 7 & \\
& Observation & 0 & 12 & \\
\hline
\end{tabular}

ASR: Additional surgical resection; SD: standard deviation; AC: adenocarcinoma; SCC: squamous cell carcinoma; ESD: diagnosis by the pathological findings of ESD specimens; $\mathrm{pT}$ tumor invasion depth from the pathological findings of surgically resected specimens; ly: lymph ductal invasion; v: vessel invasion; INF: infiltration; DI: droplet infiltration; HM: horizontal margin; VM: vertical margin; CT: chemotherapy; CRT: chemoradiotherapy.

8, another tumor that was not identified before ASR was detected based on the findings of resected specimens.

Pathological findings of ESD specimens in ASR cases with residual initial tumors and non-ASR cases with recurrence. The pathological findings of ASR cases with residual tumors and non-ASR cases with recurrence after ESD are summarized in Table III. There were no specific findings in morphology, ESD-v, ESD-HM, or ESD-VM. The tumor invasion depth of all cases was T1b, and lymph vessel invasion was observed in all cases. In all T1b cases, the detection rate of a residual initial tumor was $21.0 \%$ (4/19 cases). In all cases with positive lymph vessel invasion, the detection rate was $22.2 \%$ (4/18 cases). Moreover, 11 cases were diagnosed as $\mathrm{T} 1 \mathrm{~b}$ and with positive lymph ductal invasion, and 4 of these cases $(36.3 \%)$ had post-ESD recurrence or residual cancer.
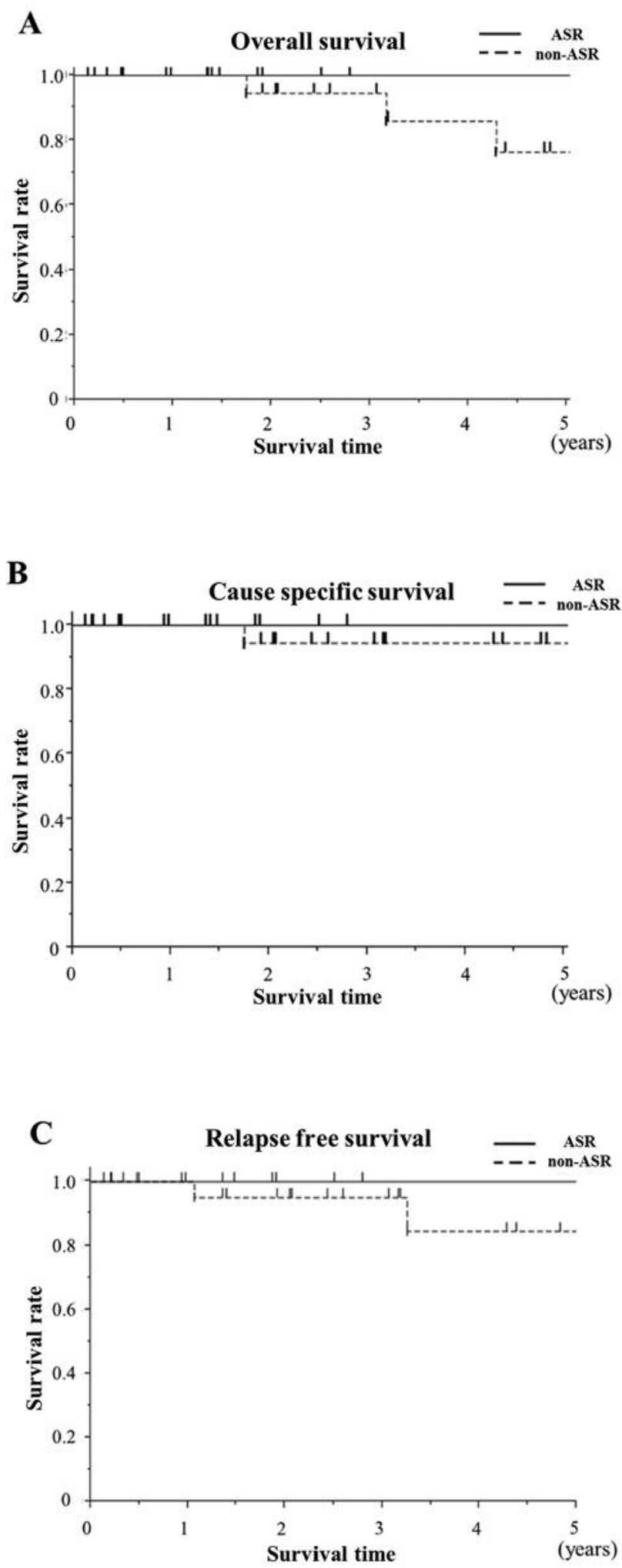

Figure 1. A: Overall survival curve of additional surgical resection (ASR) and non-additional surgical resection (non-ASR) cases. B: Causespecific survival rates of ASR and non-ASR cases. C: Relapse-free survival rates of ASR and non-ASR cases. 
Table II. Pathological findings of esophageal cancer cases that underwent additional surgical resection after ESD.

\begin{tabular}{|c|c|c|c|c|c|c|c|}
\hline Patients & Gender & Age & The reason for ASR & Histological type & Residual tumor & pT & $\mathrm{pN}$ \\
\hline Case 1 & Male & 59 & T1a-MM, ly(+) & $\mathrm{AC}$ & $(-)$ & T0 & No \\
\hline Case 2 & Male & 65 & T1a-MM, INFC & SCC & $(-)$ & T0 & No \\
\hline Case 3 & Male & 68 & T1a-MM,ly(+) & SCC & $(-)$ & T0 & No \\
\hline Case 4 & Male & 67 & T1a-MM, ly(+).INFC & SCC & $(-)$ & T0 & No \\
\hline Case 5 & Male & 54 & T1b-SM2 & SCC & $(-)$ & T0 & No \\
\hline Case 6 & Male & 77 & T1b-SM1, ly(+) & $\mathrm{AC}$ & $(+)$ & pT1a-MM & No \\
\hline Case 7 & Male & 51 & T1a-MM, VM(+) & SCC & $(-)$ & T0 & No \\
\hline Case 8 & Male & 52 & T1b-SM1, ly(+) & SCC & $(-)$ & pT1a-MM & No \\
\hline Case 19 & Male & 55 & T1a-MM, ly(+), v(+), DI (+) & SCC & $(-)$ & T0 & No \\
\hline Case 10 & Male & 57 & T1b-SM2 & SCC & $(-)$ & T0 & No \\
\hline Case 11 & Male & 62 & T1b-SM2, ly(+), v(+), DI(+) & SCC & $(-)$ & T0 & No \\
\hline Case 12 & Male & 63 & T1b-SM2, ly(+), DI(+) & SCC & $(+)$ & pT3 & No \\
\hline Case 13 & Male & 66 & T1a-MM, ly(+), v(+) & $\mathrm{AC}$ & $(-)$ & T0 & No \\
\hline Case 14 & Male & 70 & T1b-SM2, ly(+), v(+), DI(+) & SCC & $(-)$ & T0 & No \\
\hline Case 15 & Male & 72 & T1a-MM. DI(+) & SCC & $(-)$ & T0 & No \\
\hline Case 16 & Male & 72 & T1a-MM, VM(+) & $\mathrm{SCC}$ & $(-)$ & T0 & No \\
\hline
\end{tabular}

ASR: Additional surgical resection; pT: pathological invasion depth of surgically resected specimens; pN: pathological lymph node metastasis in surgically resected specimens; ly: lymph ductal invasion; v: vessel invasion; DI: droplet infiltration; AC: adenocarcinoma; SCC: squamous cell carcinoma.

Table III. Additional surgical resection cases with residual tumors after ESD, and non-additional resection cases with recurrence after ESD.

\begin{tabular}{lccccccccc}
\hline Patients & Morphology & ESD-T & ESD-ly & ESD-v & ESD-INF & ESD-HM & ESD-VM & AT & Residual tumor or recurrence \\
\hline ASR 6 & 0-IIa & pT1b-SM1 & $(+)$ & $(-)$ & INFb & $(+)$ & $(-)$ & Surgery & Res \\
ASR 12 & 0-Is+IIb & pT1b-SM2 & $(+)$ & $(-)$ & DI & $(-)$ & $(-)$ & Surgery & Res \\
non-ASR 1 & 0-IIa+IIc & pT1b-SM2 & $(+)$ & $(-)$ & Unknown & $(-)$ & $(+)$ & CT & Med-Lym \\
non-ASR 2 & 0-IIa+IIc & pT1b-SM2 & $(+)$ & $(+)$ & Unknown & $(-)$ & $(-)$ & CRT & Abd-Lym \\
\hline
\end{tabular}

ESD: Diagnosis by the pathological findings of ESD specimens; ly: lymph ductal invasion; v: vessel invasion; INF: infiltration; HM: horizontal margin; VM: vertical margin; ASR: additional surgical resection; DI: droplet infiltration; CT: chemotherapy; CRT: chemoradiotherapy; Res: residual tumor; Med-Lym: mediastinal lymph node metastasis; Abd-Lym: abdominal lymph node metastasis.

Evaluation of the surgical safety of ASR after ESD. We performed a comparative analysis of surgical safety between ASR and primary surgical resection cases (PSR). In order to unify the surgical approach in this analysis, we only analyzed cases that underwent esophagectomy with a laparoscopic transhiatal approach because we have used this approach for most superficial EC cases in recent years $(8,9)$. Twelve ASR cases and 13 PSR cases underwent esophagectomy with a laparoscopic transhiatal approach. Patient background factors, which were related to the occurrence of surgical complications, in ASR and PSR cases were summarized in Table IV. No significant differences were observed in gender, age, respiratory function, body mass index, performance status, renal disorder, hypoalbuminemia, or diabetes between ASR and PSR cases.
Table V shows the results of the comparative analysis between ASR and PSR cases for surgical time, blood loss, and the rate of surgical complications. No significant differences were observed in surgical time or blood loss. Furthermore, no significant differences were noted in the rate of surgical complications, suggesting that surgical safety of ASR was similar to that of PSR.

\section{Discussion}

Over the past decade, ESD has been widely performed for the curative treatment of several gastrointestinal cancers. While ESD was initially introduced as an EC treatment, complications, e.g. perforation, mediastinal emphysema, and esophageal stricture, and curability were limitations. 
Table IV. Background of additional surgical resection and primary surgical resection cases.

\begin{tabular}{|c|c|c|c|c|}
\hline & & $\begin{array}{c}\text { ASR } \\
(n=12)\end{array}$ & $\begin{array}{c}\text { PSR } \\
(\mathrm{n}=13)\end{array}$ & $p$-Value \\
\hline \multirow[t]{2}{*}{ Gender } & Male & 12 & 11 & \multirow[t]{2}{*}{0.156} \\
\hline & Female & 0 & 2 & \\
\hline Age & Mean $( \pm$ SD) & $65.0( \pm 7.5)$ & $60.9( \pm 10.8)$ & 0.282 \\
\hline \multicolumn{5}{|l|}{ Respiratory function } \\
\hline$\% \mathrm{VC}(\%)$ & Mean $( \pm \mathrm{SD})$ & $103.7( \pm 11.9)$ & $100.1( \pm 17.1)$ & 0.574 \\
\hline FEV1.0 (L) & Mean $( \pm$ SD $)$ & $2.8 \mathrm{~L}( \pm 0.6)$ & $2.5( \pm 0.8)$ & 0.332 \\
\hline BMI & Mean $( \pm$ SD $)$ & $20.9( \pm 2.1)$ & $22.1( \pm 5.2)$ & 0.480 \\
\hline Performance status & $1<$ & $2(16.6 \%)$ & $5(41.6 \%)$ & 0.177 \\
\hline Renal disorder & & $1(8.3 \%)$ & $0(0 \%)$ & 0.288 \\
\hline Hypoalbuminemia & & $0(0 \%)$ & $1(2.5 \%)$ & 0.327 \\
\hline Diabetes & & $0(0 \%)$ & $0(0 \%)$ & \\
\hline
\end{tabular}

ASR: Additional surgical resection; PSR: primary surgical resection; BMI: body mass index; renal disorder: $\mathrm{male}, \mathrm{Cr}>1.1 \mathrm{mg} / \mathrm{dl}, \mathrm{female}, \mathrm{Cr}>0.8$ $\mathrm{mg} / \mathrm{dl}$; hypoalbuminemia: Alb $<3.5 \mathrm{~g} / \mathrm{dl}$; diabetes: HbA1c $>6.5 \%$.

Table V. Surgical findings and complications in additional surgical resection and primary resection cases

\begin{tabular}{|c|c|c|c|c|}
\hline & & $\begin{array}{c}\text { ASR } \\
(n=12)\end{array}$ & $\begin{array}{c}\text { PSR } \\
(\mathrm{n}=13)\end{array}$ & $p$-Value \\
\hline Surgical time (min) & Mean $( \pm \mathrm{SD})$ & $285.9( \pm 57.2)$ & $238.2( \pm 81.2)$ & 0.105 \\
\hline Blood loss (ml) & Mean $( \pm \mathrm{SD})$ & $112.0( \pm 72.9)$ & $152.5( \pm 132.9)$ & 0.360 \\
\hline Leakage & & $1(7.6 \%)$ & $4(16.5 \%)$ & 0.445 \\
\hline Respiratory complications & & $3(23.0 \%)$ & $1(4.1 \%)$ & 0.077 \\
\hline Recurrent nerve paralysis & & $4(30.7 \%)$ & $6(25.0 \%)$ & 0.706 \\
\hline Surgical site infection & & $0(0 \%)$ & $3(8.1 \%)$ & 0.183 \\
\hline
\end{tabular}

ASR: Additional surgical resection; PSR: primary surgical resection; SD: standard deviation.

However, previous studies described the safety of ESD; and the indication for ESD has gradually been expanded to larger and deeper tumors $(1,2,10,11)$. In Japan, EC has been approved as an indication for ESD under health insurance coverage since 2008. The treatment outcomes of EC patients who underwent ESD have been widely reported. Furthermore, the high morbidity and mortality rates associated with conventional curative esophagectomy has accelerated the further expansion of indications for ESD. However, the 5year OS rates of superficial EC cases with tumors that were diagnosed as $\mathrm{T} 1 \mathrm{~b}$ or with positive lymphatic invasion after ESD were reported to be $71.1-77.7 \%$ and $69.8-71.0 \%$, respectively $(12,13)$. These findings suggest that some superficial EC cases need to undergo ESD as an additional treatment in order to achieve long-term survival. However, appropriate additional treatments after ESD and indications remain controversial, and clinical trials are ongoing (14).
Although indications for additional treatments after ESD have not yet been established, previous studies reported the clinical outcomes of superficial EC cases, which will contribute to its selection in clinical practice. The rates of lymph node metastasis in T1a-EP/LPM, T1a-MM, and T1b$\mathrm{SM}$ cases were previously reported to be $0 \%, 8 \%$, and $11-$ $36 \%$, respectively (15-17). Furthermore, lymph vessel invasion was identified as an independent risk factor for lymph node metastasis in T1a-MM and T1b cases (18-20). In these studies, the rates of lymph node metastasis in T1a-MM and T1b-SM1 with lymph vessel invasion were $41.7 \%$ and $100 \%$, respectively; and lymph node metastasis was not detected in T1a-MM cases without lymph vessel invasion. Meanwhile, a positive vertical margin and poor differentiation were identified as risk factors for residual tumors $(10,21)$.

Based on previous findings, ESD represents a potential curative treatment for T1a-EP, T1a-LPM, and T1a-MM EC 
cases without vessel invasion and poor differentiation. However, Sgourakis G et al. reported that the clinical diagnosis of $39 \%$ superficial EC cases was different from the pathological diagnosis after ESD (21). Therefore, difficulties are associated with selecting treatment methods against superficial EC based on an exact clinical diagnosis, and EC cases with risk factors for recurrence after ESD will continue to occur. We need to establish adequate criteria and methods for additional treatments in order to prevent the recurrence of EC.

Previous studies on additional treatments after ESD described indications and their efficacies. Motoyama $\mathrm{S}$ et al. reported that the rate of lymph node metastasis was $38.4 \%$ in $\mathrm{T} 1 \mathrm{~b}$ cases with lymph vessel invasion that underwent additional resection after ESD (22). They concluded that additional esophagectomy with lymph node dissection was effective in these cases. Ikeda A et al. performed a comparative analysis between the treatment outcomes of additional radiation/chemoradiotherapy and esophagectomy after ESD among T1a-MM or T1b cases diagnosed by the pathological findings of ESD specimens. Although OS was similar in both groups, recurrence was not observed in cases that underwent additional esophagectomy (23). Meanwhile, Kawaguchi $\mathrm{G}$ et al. reported that additional CRT after ESD was effective for T1a-MM and T1b-SM1 cases diagnosed by the pathological findings of ESD (24).

In the present study, 16 cases that underwent additional esophagectomy survived without recurrence. On the other hand, recurrence developed in $2 \mathrm{EC}$ cases that underwent non-surgical additional treatments. Although no significant differences were observed in survival curves, additional esophagectomy may be more effective than other treatments to prevent the recurrence of tumors. Two cases of recurrence after ESD were T1b-SM and had positive lymph vessel invasion. Meanwhile, a residual tumor was detected in 3 out of $16 \mathrm{EC}$ cases that underwent ASR, and these residual cases were also T1b-SM and had positive lymph vessel invasion. These results suggest that additional treatments need to be performed for T1b-SM and positive lymph vessel invasion cases, as described previously.

ESD induces inflammation in the surrounding tissues of the resected region, which may increase the difficulty of surgery, surgical time, amount of blood loss, and rate of surgical complications because of the adhesion or hardening of tissue derived from inflammation. To the best of our knowledge, a comparative analysis has not yet been conducted on the surgical safety of additional resection after ESD. Therefore, we evaluated the surgical outcomes of additional resection after ESD using a comparative analysis between ASR and PSR cases among pT1a-MM and T1b cases without lymph node metastasis. In our division, the surgical approach of esophagostomy has largely changed over the past decade. In recent years, the laparoscopic transhiatal approach has mainly been performed. In order to minimize bias in the surgical approach, we excluded EC cases that underwent surgical resection through other surgical approaches from this analysis. As a result, no significant differences were observed in surgical outcomes or the rate of complications, suggesting that the surgical safety of additional resection after ESD was similar to primary resection.

A limitation of the present study is the relatively small sample size and retrospective analysis, which may limit the statistical power. However, since evidence for additional treatments after ESD has not been established, we considered this study to provide new information on the clinical and surgical outcomes of additional resection after ESD.

In conclusion, additional treatments need to be considered for EC with T1b tumors and positive lymph vessel invasion diagnosed by the pathological findings of ESD, and additional resection is one of the effective additional treatments. The safety of additional resection after ESD was similar to primary resection for superficial EC.

\section{References}

1 Honda $\mathrm{K}$ and Akiho $\mathrm{H}$ : Endoscopic submucosal dissection for superficial esophageal squamous cell neoplasms. World J Gastrointest Pathophysiol 3: 44-50, 2012.

2 Sun F, Yuan P, Chen T and Hu J: Efficacy and complication of endoscopic submucosal dissection for superficial esophageal carcinoma: a systematic review and meta-analysis. Journal of cardiothoracic surgery 9: 78, 2014.

3 Kuwano H, Nishimura Y, Oyama T, Kato H, Kitagawa Y, Kusano M, Shimada H, Takiuchi H, Toh Y, Doki Y, Naomoto Y, Matsubara H, Miyazaki T, Muto $M$ and Yanagisawa A: Guidelines for Diagnosis and Treatment of Carcinoma of the Esophagus April 2012 edited by the Japan Esophageal Society. Esophagus 12: 1-30, 2014.

4 Egashira H, Yanagisawa A and Kato Y: Predictive factors for lymph node metastasis in esophageal squamous cell carcinomas contacting or penetrating the muscularis mucosae: the utility of droplet infiltration. Esophagus 3: 47-52, 2006.

5 Nakao R, Fujita Y, Yagi N, Konishi E and Yanagisawa A: Histopathological and immunohistochemical characteristics of superficial squamous cell carcinoma forming droplet infiltration: indicator of lymph node metastasis of the esophagus. Esophagus 12: 175-183, 2014.

6 Takubo K: Japanese Classification of Esophageal Cancer, tenth edition: part I. Esophagus 6: 1-25, 2009.

7 Takubo K: Japanese classification of esophageal cancer, tenth edition: parts II and III. Esophagus 6: 71-94, 2009.

8 Shiozaki A, Fujiwara H, Konishi H, Morimura R, Komatsu S, Murayama Y, Kuriu Y, Ikoma H, Kubota T, Nakanishi M, Ichikawa D, Okamoto K, Sakakura C and Otsuji E: Middle and lower esophagectomy preceded by hand-assisted laparoscopic transhiatal approach for distal esophageal cancer. Mol Clin Oncol 2: 31-37, 2014.

9 Fujiwara H, Shiozaki A, Konishi H, Kosuga T, Komatsu S, Ichikawa D, Okamoto $\mathrm{K}$ and Otsuji E: Single-Port Mediastinoscopic Lymphadenectomy Along the Left Recurrent Laryngeal Nerve. Ann Thorac Surg 100: 1115-1117, 2015. 
10 Joo DC, Kim GH, Park DY, Jhi JH and Song GA: Long-term outcome after endoscopic submucosal dissection in patients with superficial esophageal squamous cell carcinoma: a single-center study. Gut Liver 8: 612-618, 2014.

11 Yu JP, Liu YJ, Tao YL, Ruan RW, Cui Z, Zhu SW and Shi W: Prevention of Esophageal Stricture After Endoscopic Submucosal Dissection: A Systematic Review. World J Surg 39: 2955-2964, 2015.

12 Tachimori Y, Ozawa S, Numasaki H, Fujishiro M, Matsubara H, Oyama $\mathrm{T}$, Shinoda $\mathrm{M}$, Toh $\mathrm{Y}$ and Udagawa $\mathrm{H}$, Uno $\mathrm{T}$ : Comprehensive Registry of Esophageal Cancer in Japan, 2008. Esophagus 12: 130-157, 2015.

13 Tachimori Y, Ozawa S, Numasaki H, Fujishiro M, Matsubara H, Oyama T, Shinoda M, Toh $\mathrm{Y}$, Udagawa $\mathrm{H}$ and Uno $\mathrm{T}$ : Comprehensive Registry of Esophageal Cancer in Japan, 2007. Esophagus 12: 101-129, 2015.

14 Kurokawa Y, Muto M, Minashi K, Boku N, Fukuda H and Gastrointestinal Oncology Study Group of Japan Clinical Oncology G: A phase II trial of combined treatment of endoscopic mucosal resection and chemoradiotherapy for clinical stage I esophageal carcinoma: Japan Clinical Oncology Group Study JCOG0508. Jpn J Clin Oncol 39: 686-689, 2009.

15 Endo M, Yoshino $K$, Kawano $T$, Nagai $K$ and Inoue $H$ : Clinicopathologic analysis of lymph node metastasis in surgically resected superficial cancer of the thoracic esophagus. Dis 13: 125-129, 2000

16 Gockel I, Sgourakis G, Lyros O, Polotzek U, Schimanski CC, Lang H, Hoppo T and Jobe BA: Risk of lymph node metastasis in submucosal esophageal cancer: a review of surgically resected patients. Expert Rev Gastroenterol Hepatol 5: 371-384, 2011.

17 Cho JW, Choi SC, Jang JY, Shin SK, Choi KD, Lee JH, Kim SG, Sung JK, Jeon SW, Choi IJ, Kim GH, Jee SR, Lee WS, Jung HY and Korean ESDSG: An Endoscopist's View. Clin Endosc 47: 523-529, 2014.

18 Nakajima Y, Nagai K, Miyake S, Ohashi K, Kawano T and Iwai $\mathrm{T}$ : Evaluation of an indicator for lymph node metastasis of esophageal squamous cell carcinoma invading the submucosal layer. Japanese J Cancer Res Gann 93: 305-312, 2002.
19 Eguchi T, Nakanishi Y, Shimoda T, Iwasaki M, Igaki H, Tachimori Y, Kato H, Yamaguchi H, Saito D and Umemura S: Histopathological criteria for additional treatment after endoscopic mucosal resection for esophageal cancer: analysis of 464 surgically resected cases. Mod Pathol 19: 475-480, 2006.

20 Park JS, Youn YH, Park JJ, Kim JH and Park H: Clinical Outcomes of Endoscopic Submucosal Dissection for Superficial Esophageal Squamous Neoplasms. Clin Endosc 49: 168-175, 2016.

21 Sgourakis G, Gockel I and Lang H: Endoscopic and surgical resection of $\mathrm{T} 1 \mathrm{a} / \mathrm{T} 1 \mathrm{~b}$ esophageal neoplasms: a systematic review. World J Gastroenterol 19: 1424-1437, 2013.

22 Motoyama S, Jin M, Matsuhashi T, Nanjo H, Ishiyama K, Sato Y, Yoshino K, Sasaki T, Wakita A, Saito H, Minamiya Y, Ohnishi $\mathrm{H}$ and Ogawa $\mathrm{J}$ : Outcomes of patients receiving additional esophagectomy after endoscopic resection for clinically mucosal, but pathologically submucosal, squamous cell carcinoma of the esophagus. Surg Today 43: 638-642, 2013.

23 Ikeda A, Hoshi N, Yoshizaki T, Fujishima Y, Ishida T, Morita Y, Ejima Y, Toyonaga T, Kakechi Y, Yokosaki H and Azuma T: Endoscopic Submucosal Dissection (ESD) with Additional Therapy for Superficial Esophageal Cancer with Submucosal Invasion. Internal Medicine 54: 2803-2813, 2015.

24 Kawaguchi G, Sasamoto R, Abe E, Ohta A, Sato H, Tanaka K, Maruyama K, Kaizu M, Ayukawa F, Yamana N, Liu J, Takeuchi M, Kobayashi M and Aoyama H: The effectiveness of endoscopic submucosal dissection followed by chemoradiotherapy for superficial esophageal cancer. Radiat Oncol 10: 31, 2015.

Received July 6, 2017

Revised August 3, 2017

Accepted August 4, 2017 